\title{
A Note on the Normal Index and the $c$-Section of Maximal Subgroups of a Finite Group
}

\author{
Na Tang ${ }^{1,2}$ and Xianhua $\mathrm{Li}^{1}$ \\ ${ }^{1}$ School of Mathematical Science, Soochow University, Suzhou 215006, China \\ ${ }^{2}$ School of Mathematical Science, Huaiyin Normal University, Huai'an 223300, China \\ Correspondence should be addressed to Na Tang; hytn999@126.com
}

Received 21 April 2014; Accepted 15 July 2014; Published 22 July 2014

Academic Editor: Junjie Wei

Copyright (C) 2014 N. Tang and X. Li. This is an open access article distributed under the Creative Commons Attribution License, which permits unrestricted use, distribution, and reproduction in any medium, provided the original work is properly cited.

Let $M$ be a maximal subgroup of finite group $G$. For each chief factor $H / K$ of $G$ such that $K \leq M$ and $G=M H$, we called the order of $H / K$ the normal index of $M$ and $(M \cap H) / K$ a section of $M$ in $G$. Using the concepts of normal index and $c$-section, we obtain some new characterizations of $p$-solvable, 2 -supersolvable, and $p$-nilpotent.

\section{Introduction}

In this paper, all groups considered are finite. Let $\pi(G)$ denote the set of prime divisors of $|G|$, and for $p \in \pi(G) \operatorname{let} \operatorname{Syl}_{p}(G)$ denote the set of Sylow $p$-subgroups of $G$. Write $M \lessdot G$ to indicate that $M$ is a maximal subgroup of $G$. For convenience, we cite the following relative definitions. For a fixed prime $p \in \pi(G)$,

(1) $\mathscr{F}_{c}(G)=\{M \mid M \lessdot G$ and $|G: M|$ is composite $\}$,

(2) $\mathscr{F}_{p}(G)=\left\{M \mid M \lessdot G\right.$ and $\left.|G: M|_{p}=1\right\}$,

(3) $\left.\mathscr{F}_{p c}(G)=\mathscr{F}_{p}(G) \cap \mathscr{F}_{c}(G)\right\}$,

(4) $\mathscr{F}^{P}(G)=\left\{M \mid M \lessdot G\right.$ and $\left.N_{G}(P) \leq M\right\}$, where $P \in \operatorname{Syl}_{p}(G)$

(5) $\mathscr{F}^{p c}(G)=\mathscr{F}^{p}(G) \cap \mathscr{F}_{c}(G)$.

The remaining notation and terminology in this paper are standard, as in Huppert [1].

In 1959, Deskins [2] introduced the concept of normal index. For a maximal subgroup $M$ of a group $G$, the order of a chief factor $H / K$ of $G$, where $H$ is minimal in the set of normal supplements of $M$ in $G$, is known as the normal index of $M$ of $G$, denoted by $\eta(G: M)$. If $H / K$ is such a chief factor, then $G=M H, K \leq M$, and $|G: M|=$ $|H / K:(H / K) \cap(M / K)|$, so $|H / K|=|(H \cap M) / K \| G: M|$.
The intersection $(M \cap H) / K$ is called a $c$-section of $M$. Li and Wang in [3] proved that every maximal subgroup $M$ of $G$ has a unique $c$-section up to isomorphism. Let $\operatorname{Sec}(M)$ denote a group which is isomorphic to a $c$-section of $M$. Then $\eta(G: M)=|\operatorname{Sec}(M)| \cdot|G: M|$. Deskins [2] showed that $G$ is solvable if and only if $\eta(G: M)=|G: M|$ for every maximal subgroup $M$ of $G$. The investigations on the normal index have been developed by many scholars; see [3-7]. But the earlier results concern the cases where $p$ is either the largest prime dividing $|G|$ or an odd prime. In 2010, Zhang and $\mathrm{Li}$ analyzed the case when $p=2$ and obtained some interesting results. In particular we note the following theorems.

Theorem 1 (see [8, Theorem 3.1]). A group $G$ is solvable if and only if $\eta(G: M)_{2}=1$ for every $M \in \mathscr{F}^{2}(G)$.

Theorem 2 (see [8, Theorem 3.4]). A group G is solvable if and only if $\operatorname{Sec}(M)$ is either a 2'-group or an abelian 2-group for every $M \in \mathscr{F}^{2}(G)$.

We observe that Theorems 1 and 2 still hold by replacing 2 with another prime $p$. For example, let $G=S_{4}$ and let $M \in$ $\mathscr{F}^{3}(G)$. Since the order of $M$ is 6 or $12, \eta(G: M)_{3}=1$. So $G$ satisfies the hypotheses of Theorem 1. But $G$ is 3-solvable. It is natural to ask that the theorems above hold or not for any prime $p$. In part 3 , we give positive answer and relative results. 


\section{Preliminary Results}

Lemma 3 (see [8, Lemma 2.2]). Let $G$ be a group, $N$ a normal subgroup of $G$, and $p \in \pi(G)$. Let $M$ be a maximal subgroup of $G$ and $N \leq M$.

(1) We have $\eta(G / N: M / N)=\eta(G: M)$ and $\operatorname{Sec}(M / N) \cong \operatorname{Sec}(M)$.

(2) If $M / N \in \mathscr{F}_{p}(G / N)$, then $M \in \mathscr{F}_{p}(G)$.

(3) If $M / N \in \mathscr{F}^{p}(G / N)$, then $M \in \mathscr{F}^{p}(G)$.

(4) If $p=\max \pi(G)$, then $\mathscr{F}^{p}(G)=\mathscr{F}^{p c}(G)$.

Lemma 4 (see [6, Theorem 7]). G is p-supersolvable if and only if, for each maximal subgroup $M$ of $G, \eta(G: M)_{p}=\mid G$ : $\left.M\right|_{p}=1$ or $p$.

\section{Main Results}

Theorem 5. $G$ is $p$-solvable if and only if $\eta(G: M)_{p}=1$ for every $M \in \mathscr{F}^{P}(G)$.

Proof. $\Rightarrow$ : Suppose that $G$ is $p$-solvable and let $N$ be a minimal normal subgroup. If a maximal subgroup $M \in$ $\mathscr{F}^{p}(G)$ containing $N$, then, by induction, it follows that $\eta(G / N: M / N)_{p}=\eta(G: M)_{p}=1$. If $N \nsubseteq M$, then we must have $|N|_{p}=1$, since $N$ is a $p^{\prime}$-group.

$\Leftarrow$ : Conversely, let $\eta(G: M)_{p}=1$ hold for each maximal subgroup $M \in \mathscr{F}^{2}(G)$. Only we need to consider that $G$ is not simple. Otherwise, $|G|_{p}=\eta(G: M)_{p}=1$. Certainly, $G$ is p-solvable.

Now let $N$ be a minimal normal subgroup of $G$. Observe the quotient group $G / N$. For every maximal subgroup $M / N \in \mathscr{F}^{P}(G / N)$, it is easy to see $M \in \mathscr{F}^{P}(G)$. By Lemma 3 and hypothesis, $\eta(G / N: M / N)_{p}=\eta(G: M)_{p}=1$. Hence $G / N$ is $p$-solvable by induction. Since the class of all $p$ solvable groups is a saturated formation, we may suppose that $N$ is the unique minimal normal subgroup of $G$. If $|N|_{p}=1$, then $N$ is a $p^{\prime}$-group. Moreover, $G / N$ is $p$-solvable, and so is $G$. Now consider $|N|_{p} \neq 1$. Let $P$ be a Sylow $p$-subgroup of $G$ and $K=P \cap N$. Then $K$ is a Sylow $p$-subgroup of $N$. Clearly, $N_{G}(P) \leq N_{G}(K)<G$. So $N_{G}(K)$ is contained in some maximal subgroup $T$ of $G$. Hence $T \in \mathscr{F}^{P}(G)$. By Frattini argument, $G=N N_{G}(K)=N T$. It follows that $|N|_{p}=\eta(G: T)_{p}=1$, a contradiction, and we are done.

Corollary 6. $G$ is solvable if and only if, for every $M \in \mathscr{F}^{p}(G)$, $\eta(G: M)=1$, where $p$ is an arbitrary divisor of $|G|$.

It was announced by Zhang and Li in [8, Theorem 5] that a group $G$ is solvable if and only if $\operatorname{Sec}(M)$ is a $2^{\prime}$-group or an abelian 2-group for $M \in \mathscr{F}^{2}(G)$. We extend this theorem by proving the following.

Theorem 7. $G$ is p-solvable if and only if, for any $M \in \mathscr{F}^{p}(G)$, $\operatorname{Sec}(M)$ is an abelian p-group or a $p^{\prime}$-group, where $p$ is a prime divisor of $|G|$.
Proof. $\Rightarrow$ : Suppose that $G$ is $p$-solvable and let $N$ be a minimal normal subgroup. If a maximal subgroup $M \epsilon$ $\mathscr{F}^{p}(G)$ containing $N$, then, by induction, it follows that $\operatorname{Sec}(M / N) \cong \operatorname{Sec}(M)$ is an abelian $p$-group or a $p^{\prime}$-group in view of Lemma 3. If $N \nsubseteq M$, then $G=M N$. If $|N|_{p}=1$, then $\eta(G: M)_{p}=|N|_{p}=1$, and $\operatorname{so} \operatorname{Sec}(M)$ is a $p^{\prime}$ group. Now consider $|N|_{p}=1$. By the $p$-solvability of $G$, it implies that $N$ is an elementary abelian $p$-group. It follows that $\operatorname{Sec}(M) \cong M \cap N$ is an abelian $p$-group.

$\Leftarrow$ : Conversely, suppose $\operatorname{Sec}(M)$ is an abelian $p$-group or a $p^{\prime}$-group. Let $N$ be a minimal normal subgroup of $G$. By Lemma 3, $G / N$ satisfies the hypotheses of the theorem. Then by induction, $G / N$ is $p$-solvability. If $|N|_{p}=1$, then $G$ is $p$ solvable. Now assume that $|N|_{p} \neq 1$, then $G$ is $p$-solvable. Let $P$ be a Sylow $p$-solvable of $G$ and $K=P \cap N$. Then, $K$ is a Sylow $p$-subgroup of $N$. Obviously, $N_{G}(P) \leq N_{G}(K)<G$. So $N_{G}(K)$ is contained in some maximal subgroup $T$ of $G$, and consequently, $T \in \mathscr{F}^{P}(G)$. By Frattini argument, $G=$ $N N_{G}(K)=N T$. Then the minimal normality of $N$ shows $\operatorname{Sec}(M) \cong M \cap N$. On the other hand, $K \leq N_{N}(K) \leq M \cap N$. Combining the hypothesis, $\operatorname{Sec}(M)$ is an abelian $p$-group, and so is $M \cap N$. It follows that $N_{N}(K)=C_{N}(K)$. By Burnside Theorem, $N$ is $p$-nilpotent, which contradicts the minimal normality of $N$. Therefore, the conclusion holds.

In view of Theorem 7 it is natural to ask if a group $G$ is $p$-solvable when $|\operatorname{Sec}(M)|_{p}=p^{\alpha}$ or 1 , for $M \in \mathscr{F}^{p}(G)$, where $p$ is a prime divisor of $|G|$. The answer of the question is negative. For example, set $G=\operatorname{PSL}(2,7)$ and $p=3$; every maximal subgroup $M$ satisfies that $|\operatorname{Sec}(M)|_{3}=3$, but $G$ is not 3-solvable. For $p$-solvable, the condition that $\operatorname{Sec}(M)$ is an abelian $p$-group is crucial.

It is proved in [6, Theorem 7] that a group $G$ is $p$ supersolvable if and only if, for each maximal subgroup $M$ of $G, \eta(G: M)_{p}=|G: M|_{p}=1$ or $p$. It is natural to ask if a group $G$ is $p$-supersolvable when $\eta(G: M)_{p}=1$ or $p$ for any maximal subgroup $M$ of $G$. The answer of the question is negative. For example, set $G=\operatorname{PSL}(2,7)$ and $p=3$; every maximal subgroup $M$ satisfies that $\eta(G: M)_{3}=3$, but $G$ is not 3-supersolvable. But assuming that $p=2$, the result holds or not. For the question, we give the positive answer. Next, we prove the result.

Theorem 8. $G$ is 2-supersolvable if and only if, for any maximal subgroup $M$ of $G, \eta(G: M)_{2}=1$ or 2 .

Proof. $\Rightarrow$ : Suppose that $G$ is 2-supersolvable. Certainly, $G$ is solvable. By Lemma 4, the necessity holds.

$\Leftarrow$ : Conversely, assume the result is not true and let $G$ be a counterexample of minimal order. Now, we assert $G$ is not simple. If not, then $\eta(G: M)_{2}=|G|_{2}=1$ or 2 . For $|G|_{2}=1$, it is clear that $G$ is 2 -supersolvable, a contradiction. Assume that $|G|_{2}=2$. Then $G$ is a cyclic group of order 2 , and so $G$ is 2-supersolvable, a contradiction. This contradiction shows $G$ is not simple. Let $N$ be the minimal normal subgroup of $G$. By Lemma 3, G/N satisfies the hypotheses of the theorem. The minimal choice of $G$ implies that $G / N$ is 2-supersolvable. If $N$ is contained in each maximal subgroup $M$ of $G$, then $N \subseteq \Phi(G)$, and consequently, $G / \Phi(G)$ is 2-supersolvable, and 
so is $G$, a contradiction. Hence there is a maximal subgroup $M$ of $G$, such that $G=M N$. Suppose that $|N|_{2}=1$. It follows that $G$ is 2-supersolvable, a contradiction. So $|N|_{2} \neq 1$. By hypothesis, $\eta(G: M)_{2}=|N|_{2}=2$. Moreover, $N$ is solvable. Therefore, $|N|=2$, and so $G$ is 2-supersolvable, which contradicts the assumption. Now the proof of theorem is completed.

Theorem 9. Suppose $G$ is a group and $p$ is the smallest prime divisor of $|G|$. Then $G$ is p-nilpotent if and only if the following conditions are satisfied:

(1) $\eta(G: M)_{p}=1$ or $p$ for every maximal subgroup $M$ of G;

(2) if $\eta(G: M)_{p}=p$ for some maximal subgroup $M$, then $M \unlhd G$.

Proof. $\Rightarrow$ : Assume that $G$ is $p$-nilpotent. Then $G$ is $p$ supersolvable and (1) holds by Lemma 4 . Now let $M$ be a maximal subgroup of $G$ with $\eta(G: M)_{p}=p$ and $G=P T$, where $P$ is a Sylow $p$-subgroup and $T$ is a normal Hall $p^{\prime}$ subgroup of $G$. Suppose $T \nsubseteq M$ and let $1 \unlhd \cdots \unlhd T_{2} \unlhd T_{1} \unlhd$ $\cdots \unlhd T \unlhd G$ be a chief group series, where $T_{1} \npreceq M$ and $T_{2} \leq M$. Then $\eta(G: M)_{p}=\left|T_{1} / T_{2}\right|_{p}=1$, a contradiction. Hence $T \subseteq M$. Since $\eta(G: M)_{p}=p,|G: M|_{p}=1$ or $p$. If $|G: M|_{p}=1$, then some Sylow $p$-subgroup of $G$, say $P_{1}$, is contained in $M$, and it follows that $G=P_{1} T \subseteq M$, a contradiction. Therefore, $|G: M|_{p}=p$. Since $M=T(M \cap P)$, $|P| /|M \cap P|=|G: M|_{p}=p$, which leads to $P \cap M \unlhd P$. Hence $M \unlhd P M=G$.

$\Leftarrow$ : Now suppose (1) and (2) hold. If, for each maximal subgroup $M$ of $G, \eta(G: M)_{p}=1$, then by Theorem $8, G$ is $p$-solvable. Combining condition (2), we have that $G$ is not simple. Let $N$ be a minimal normal subgroup of $G$. By Lemma 3, $G / N$ satisfies the hypotheses. By induction, $G / N$ is $p$-nilpotent. Since the class of all $p$-nilpotent groups is a saturated formation, we may regard $N$ as the unique minimal normal subgroup of $G$ and $\Phi(G)=1$. So there exists a maximal subgroup $M$ of $G$ such that $G=N M$ and $\eta(G$ : $M)_{p}=|N|_{p}=1$ or $p$.

Suppose $|N|_{p}=1$. Then $N$ is a $p^{\prime}$-group. Since $G / N$ is $p$-nilpotent, $G$ is $p$-nilpotent.

Assume $|N|_{p}=p$. Since $p$ is the smallest prime divisor of $|G|, N$ is $p$-nilpotent, and so $|N|=p$. It now follows that $M \cap N=1$ and $M \cong G / N$ is $p$-nilpotent. Note $M=M_{p} M_{p^{\prime}}$, where $M_{p}$ is a Sylow $p$-subgroup and $M_{p^{\prime}}$ is a normal Hall $p^{\prime}$-subgroup of $M$. Then by (2), $M_{p^{\prime}}$ char $M \unlhd G$, and so $M_{p^{\prime}} \unlhd G$. Consequently, $G=M N=\left(M_{p} N\right) M_{p^{\prime}}$ and $M_{p^{\prime}}$ is a normal Hall $p^{\prime}$-subgroup of $G$.

The proof of the theorem has been done.

Obviously, in Theorem 9, removing the condition " $p$ is the smallest prime divisor of $|G|$ ", and the result does not hold.

Theorem 10. G has a p-nilpotent maximal subgroup $M$ with prime power normal index; then $G$ is p-solvable.

Proof. Assume that the theorem is false and let $G$ be a minimal counterexample. Let $M$ be a $p$-solvable maximal subgroup of $G$ with $\eta(G: M)=q^{\alpha}$, where $q$ is a prime. Now we assert that $G$ is not simple. Otherwise, $\eta(G: M)=|G|=$ $q^{\alpha}$, a contradiction. Let $N$ be a minimal normal subgroup of $G$. Next, we consider the following two cases.

Case $1(N \subseteq M)$. Then by Lemma 3, $\eta(G / N: M / N)=\eta(G$ : $M)=q^{\alpha}$. Since $M$ is $p$-solvable, $M / N$ and $N$ are $p$-solvable. By the minimal choice of $G$, it implies that $G / N$ is $p$-solvable, so is $G$, a contradiction.

Case $2(N \nsubseteq M)$. Then $G=M N$ and $G / N \cong M /(M \cap N)$ is $p$-solvable. On the other hand, $\eta(G: M)=|N|$ is a $q$-group. Thus $G$ is $p$-solvable, a final contradiction. This contradiction completes the proof of the theorem.

Theorem 11. If $G$ has a $p$-supersolvable maximal subgroup $M$ such that $\eta(G: M)$ is a prime and $M_{G}=1$, then $G$ is $p$ supersolvable.

Proof. Assume the result is not true and let $G$ be a counterexample of minimal order. By Theorem 10, $G$ is $p$-solvable. Let $N$ be an arbitrary minimal normal subgroup of $G$. Then $N$ is an abelian $p$-group or a $p^{\prime}$-group. Moreover, since $M_{G}=1, G=M N$. Suppose every minimal normal subgroup $N$ of $G$ is a $p^{\prime}$-group. But $G / N=M N / N \cong M / M \cap$ $N$ is $p$-supersolvable; it follows that $G$ is $p$-supersolvable, a contradiction. This contradiction shows that there exists some minimal normal subgroup $K$ of $G$ which is an abelian $p$-group. Then $G=M K$ and $M \cap K=1$. From this it follows that $\eta(G: M)=|K|$ is a prime. Since $G / K \cong M$ is $p$ supersolvable, $G$ is $p$-supersolvable, a contradiction. Hence the result holds.

\section{Conflict of Interests}

The authors declare that there is no conflict of interests regarding the publication of this paper.

\section{Acknowledgments}

Research of the authors is supported by NNSF Grant of China (Grants 11171243 and 11001098), Natural Science Foundation of Jiangsu (Grant BK20140451), and University Natural Science Foundation of Jiangsu (Grant 14KJB110002). The authors thank the referees and editors for their many valuable comments and suggestions.

\section{References}

[1] B. Huppert, Endliche Gruppen I, vol. 134 of Die Grundlehren der Mathematischen Wissenschaften, Springer, Berlin, Germany, 1967.

[2] W. E. Deskins, "On maximal subgroups," in Finite Groups: Proceedings of a Symposium in Pure Mathematics of the American Mathematical Society, vol. 1 of Proceedings of Symposia in Pure Mathematics, pp. 100-104, American Mathematical Society, 1959.

[3] S. Li and Y. Wang, "On $C$-section and $C$-index of finite groups," Journal of Pure and Applied Algebra, vol. 151, no. 3, pp. 309-319, 2000 . 
[4] A. Ballester-Bolinches, "On the normal index of maximal subgroups in finite groups," Journal of Pure and Applied Algebra, vol. 64, no. 2, pp. 113-118, 1990.

[5] J. C. Beidleman and A. E. Spencer, "The normal index of maximal subgroups in finite groups," Illinois Journal of Mathematics, vol. 16, pp. 95-101, 1972.

[6] N. P. Mukherjee, "A note on normal index and maximal subgroups in finite groups," Illinois Journal of Mathematics, vol. 19, no. 2, pp. 173-178, 1975.

[7] N. P. Mukherjee and P. Bhattacharya, "The normal index of a finite group," Pacific Journal of Mathematics, vol. 132, no. 1, pp. 143-149, 1988.

[8] X. Zhang and X. Li, "On the normal index and the $c$-section of maximal subgroups of a finite group," Journal of Group Theory, vol. 13, no. 3, pp. 447-457, 2010. 


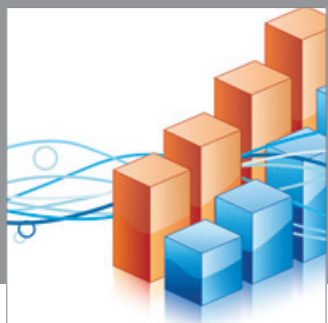

Advances in

Operations Research

mansans

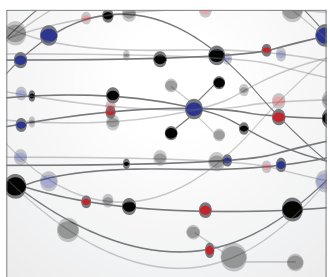

The Scientific World Journal
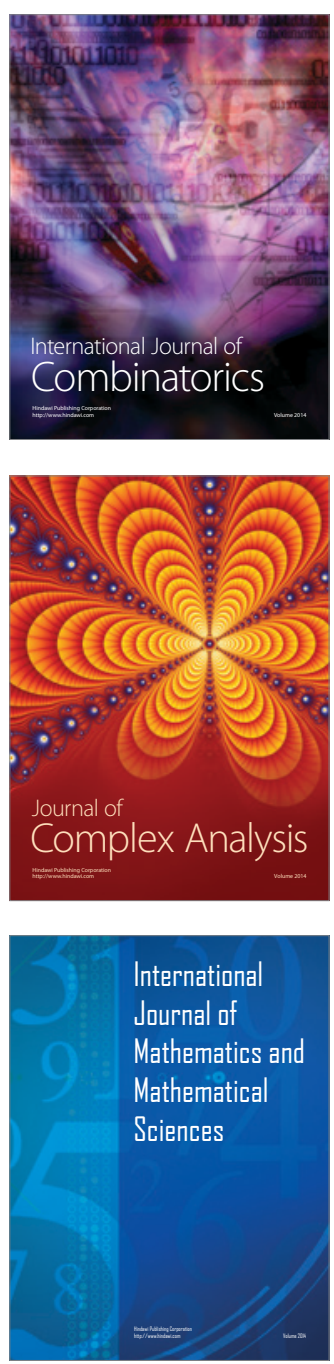
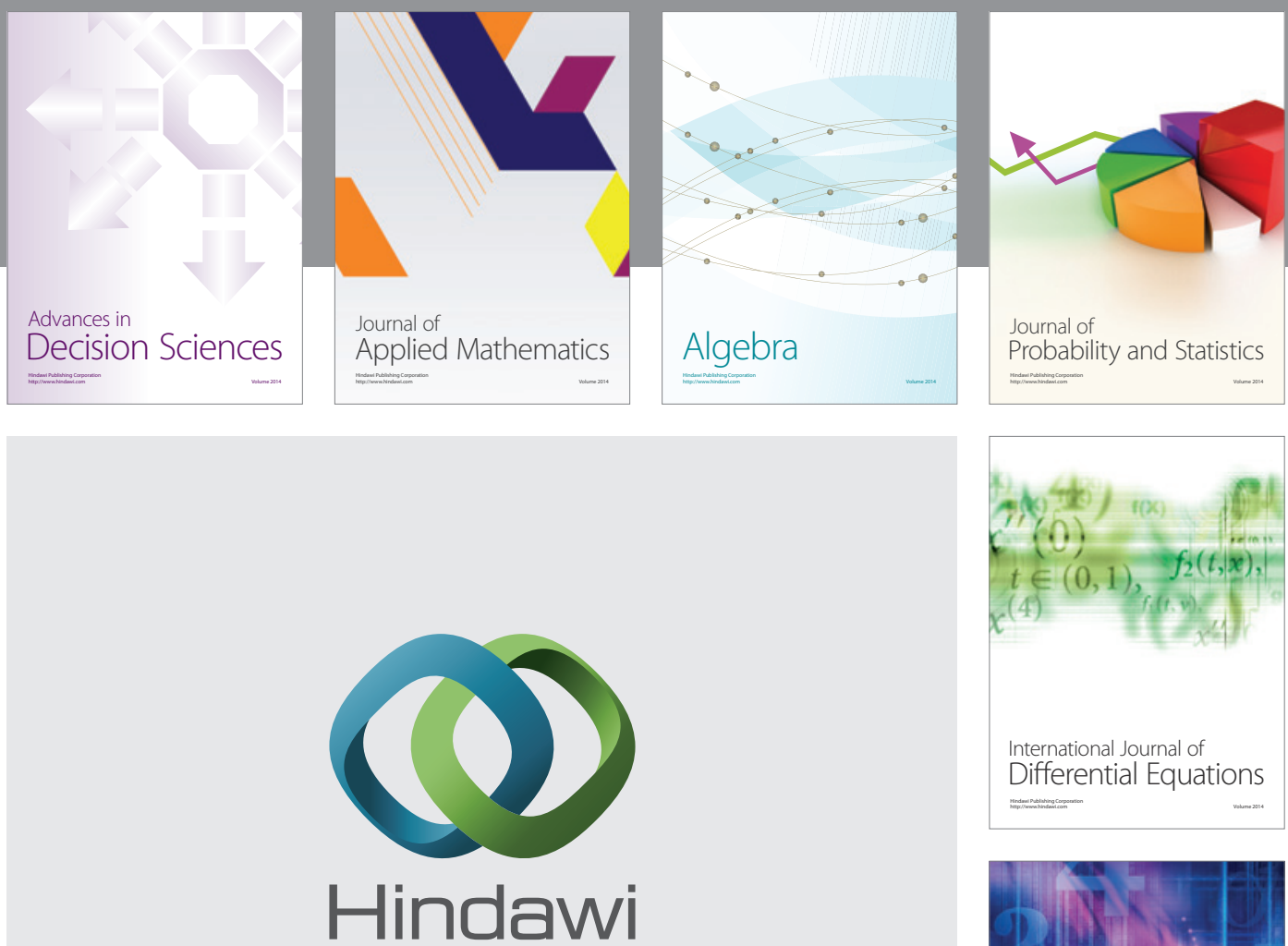

Submit your manuscripts at http://www.hindawi.com
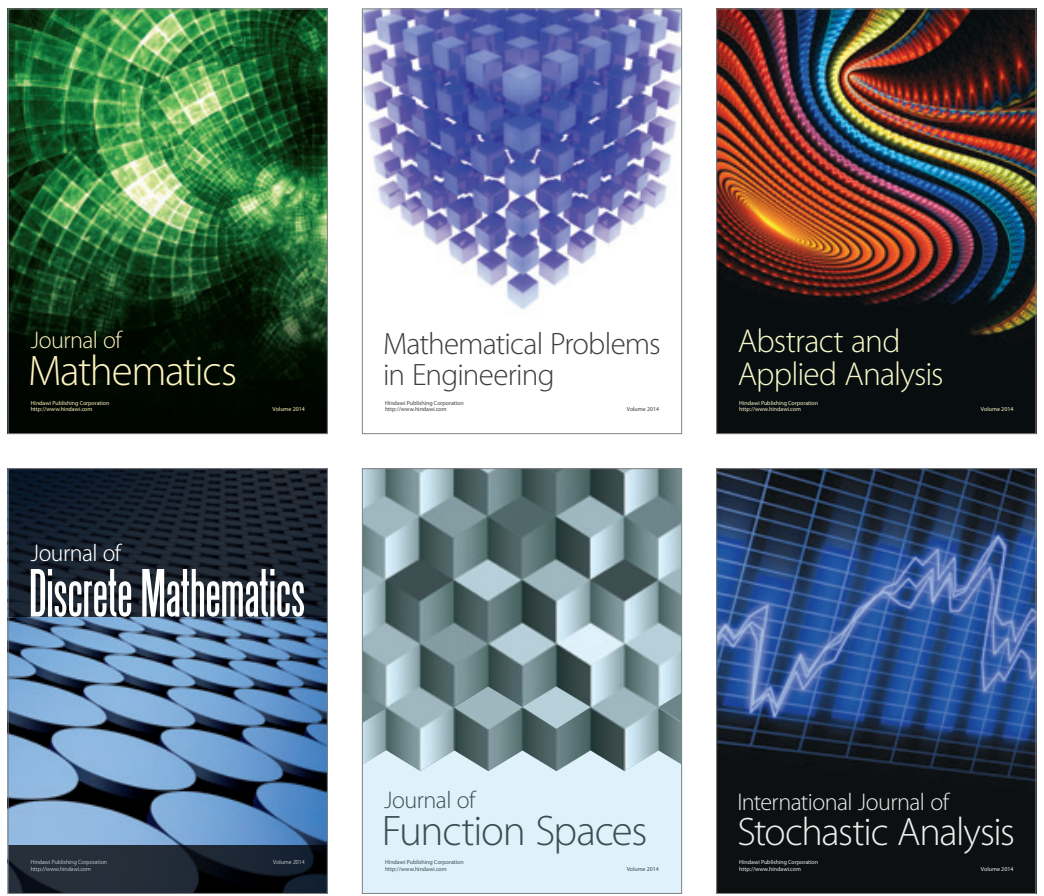

Journal of

Function Spaces

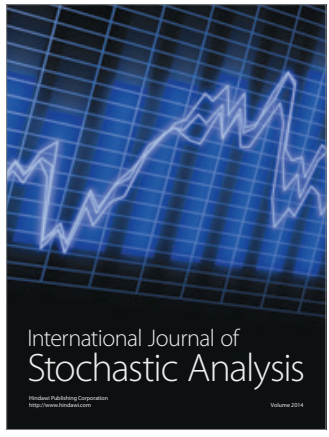

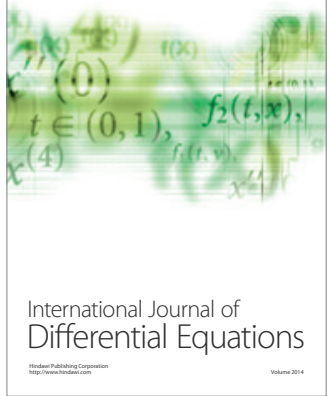
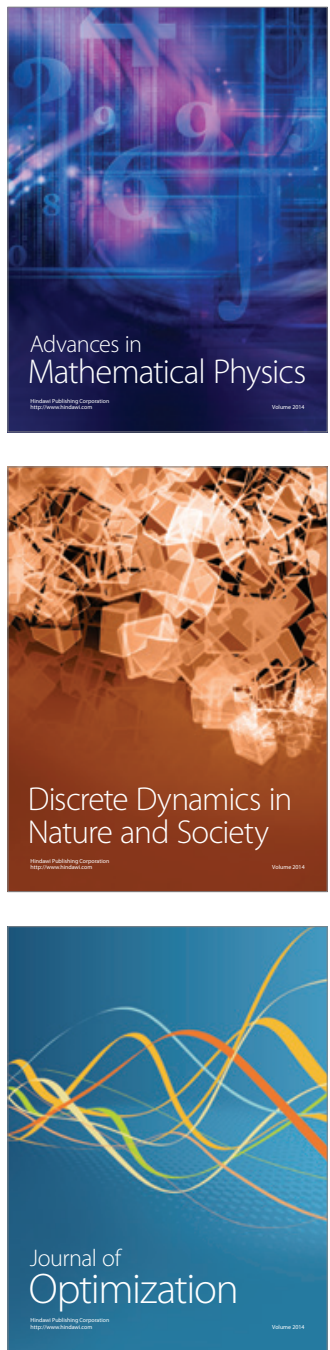